\title{
Enhanced expression of PKM2 associates with the biological properties of cancer stem cells from A549 human lung cancer cells
}

\author{
CHANG-YING GUO ${ }^{1-3}$, CHEN YAN $^{3}$, LAN LUO $^{3}$, SHINJI GOTO $^{3}$, YOSHISHIGE URATA ${ }^{3}$, \\ JIAN-JUN XU ${ }^{1}$, XIAO-MING WEN ${ }^{2}$, YU-KANG KUANG ${ }^{2}$, FANG-FANG TOU ${ }^{2}$ and TAO-SHENG LI ${ }^{3}$ \\ ${ }^{1}$ Department of Thoracic Surgery, Second Affiliated Hospital of Nanchang University, Nanchang, Jiangxi 330006; \\ ${ }^{2}$ Department of Thoracic Surgery, Jiangxi Provincial Cancer Hospital, Nanchang, Jiangxi 330029; \\ ${ }^{3}$ Department of Stem Cell Biology, Nagasaki University Graduate School of Biomedical Sciences, \\ Nagasaki University, Nagasaki 852-8523, Japan
}

Received September 8, 2016; Accepted January 25, 2017

DOI: $10.3892 /$ or.2017.5438

\begin{abstract}
Cancer cells express the M2 isoform of glycolytic enzyme pyruvate kinase (PKM2) for favoring the survival under a hypoxic condition. Considering the relative low oxygen microenvironment in stem cell niche, we hypothesized that an enhanced PKM2 expression associates with the biological properties of cancer stem cells. We used A549 human lung cancer cell line and surgical resected lung cancer tissue samples from patients for experiments. We confirmed the co-localization of PKM2 and CD44, a popular marker for cancer stem cells in lung cancer tissue samples from patients. The expression of PKM2 was clearly observed in approximately $80 \%$ of the A549 human lung cancer cells. Remarkably, enhanced expression of PKM2 was specially observed in these cells that also positively expressed CD44. Downregulation of PKM2 in $\mathrm{CD} 44^{+}$cancer stem cells by siRNA significantly impaired the potency for spheroid formation, decreased the cell survival under fetal bovine serum deprivation and hypoxic conditions, but increased their sensitivity to anti-cancer drug of cisplatin and $\gamma$-ray. The enhanced expression of PKM2 seems to associate with the biological properties of cancer stem cells from A549 human lung cancer cells. Selective targeting of PKM2 may provide a new strategy for cancer therapy, especially for patients with therapeutic resistance.
\end{abstract}

\section{Introduction}

Pyruvate kinase converts phosphoenolpyruvate to pyruvate, catalyzing the rate-limiting step of glycolysis (1). The M1 isoenzyme of pyruvate kinase (PKM1) is found in adult tissues, but the M2 isoenzyme of pyruvate kinase (PKM2) is

Correspondence to: Professor Tao-Sheng Li, Department of Stem Cell Biology, Atomic Bomb Disease Institute, Nagasaki University, 1-12-4 Sakamoto, Nagasaki 852-8523, Japan

E-mail: litaoshe@nagasaki-u.ac.jp

Key words: PKM2, CD44, cancer stem cells, resistance, neoplasms a spliced variant found in embryonic stem cells (2) and cancer cells $(3,4)$. It has been recently demonstrated that expression of PKM2 contributes to maintain the pluripotency of embryonic stem cells under hypoxic condition (2). The expression of PKM2 in cancer cells is well known to shift an aerobic glycolysis, a phenomenon known as Warburg effect (3-5). Beyond the metabolic function, PKM2 expression in cancer cells is also well known to regulate tumor formation and growth by being instrumental in gene transcription $(6,7)$. Many studies have demonstrated that the enhanced expression of PKM2 associates with therapeutic resistance and contributes to poor prognosis of cancers $(8,9)$. However, it is still necessary to further understand how PKM2 regulates the cell biological property of cancer cells.

The heterogeneity of cancer cells is generally accepted, and a stem cell-like subpopulation, known as 'cancer stem cells' (CSCs) has been identified in various types of malignant tumors $(10,11)$. Although lacking of consensus on the definition and markers for identification, CSCs are widely recognized as a subpopulation among cancer cells that keeps the properties of self-renewal and tumor initiation $(10,11)$. CSCs are also well known to play key roles in the tumor therapeutic resistance, metastasis and recurrence (12). Therefore, CSCs are considered to be promising targets for the treatment of cancers.

Normal tissue specific stem cells are considered as the main origin of cancers $(13,14)$, and the CSCs are thought to have inherited, at least partly, the characterization of normal tissue specific stem cells. Actually, the identification of CSCs has simply shared markers of hematopoietic stem cells, including the most popularly used cell surface markers of CD44 and CD133 (15-17). The expression of either CD44 or CD133 in cancer cells has also been previously demonstrated to contribute to therapeutic resistance $(18,19)$. As CD44 positive lung cancer cells were shown to have stem-like properties in a previous study (17), we used CD44 as lung cancer stem cell biomarker in the present study. Considering the relatively low oxygen microenvironment of stem cell niche $(20,21)$, we herein examined the hypothesis that cancer cells might enhance the expression of glycolytic enzyme PKM2 to facilitate the phenotype of CSCs, which thereby change the cell biological 
properties in resistance to chemotherapy, radiotherapy and various stresses.

\section{Materials and methods}

Immunohistological analysis on the expression of PKM2 and CD44 in human lung cancer tissues. By using surgical resected tissue samples from 5 patients of lung cancer (3 slides per sample), we investigated the expression and co-localization of PKM2 and CD44 in lung cancer cells. Briefly, 5- $\mu \mathrm{m}$ paraffin tissue sections were mounted on polylysine-coated slides. The slides were deparaffinized in xylene, cleared in a graded ethanol series and heat-treated for $30 \mathrm{~min}$ in $10 \mathrm{mM}$ citrate buffer $\mathrm{pH} 7.0$ in a microwave at $98^{\circ} \mathrm{C}$ for antigen retrieval. After blocking with Blocking One Histo (Nacalai Tesque, Inc., Kyoto, Japan), slides were stained with primary antibody specific against PKM2 (\#4053S; Cell Signal Technology, Danvers, MA, USA) for $1 \mathrm{~h}$ at room temperature, and then followed by PE-conjugated secondary antibody. Slides were washed and then incubated with FITC-conjugated rabbit anti-human CD44 antibody (R\&D Systems, Minneapolis, MN, USA). Nuclei were labelled with DAPI. Informed consent was obtained from each patient, and the study protocol was approved by the Ethics Committee of Jiangxi Cancer Hospital.

Human lung cancer cells and cell culture. The A549 human lung cancer cells were maintained in RPMI-1640 basic medium supplemented with $10 \%$ fetal bovine serum (FBS), in a humidified atmosphere of $95 \%$ air and $5 \% \mathrm{CO}_{2}$ at $37^{\circ} \mathrm{C}$.

Immunocytochemistry. To confirm the relationship between an enhanced expression of PKM2 and the phenotype of CSCs, we performed double immunostaining with PKM2 and CD44. Briefly, cells cultured on 4-well culture slides were fixed in $1 \%$ formaldehyde for $10 \mathrm{~min}$. After blocking with $2 \%$ bovine serum albumin (BSA), the cells were incubated with the rabbit specific anti-human PKM2 polyclonal antibody (\#4053S; Cell Signal Technology) for $1 \mathrm{~h}$ at room temperature and then followed by PE-conjugated secondary antibody. Culture slides were washed and then were incubated with FITC-conjugated rabbit anti-human CD44 antibody (R\&D Systems). Cell nuclei were stained with DAPI. The positively stained cells were observed under fluorescence microscope with 200 -fold magnification.

The purification of $\mathrm{CD} 44^{+} \mathrm{CSCs}$. The $\mathrm{CD} 44^{+} \mathrm{CSCs}$ were purified by using the Magnetic Cell Sorting system (autoMACS; Miltenyi Biotec, Inc., Auburn, CA, USA) (22). Briefly, single cell suspension of A549 cells was incubated with anti-human CD44 antibody (Miltenyi Biotec) for $30 \mathrm{~min}$. After washing, $\mathrm{CD} 44^{+}$cells were separated by passing a MACS column. The purity of the CD44+ ${ }^{+}$CSCs collected by the autoMACS was $\sim 95 \%$.

Knockdown of PKM2. To further understand the biological roles of PKM2 expression in cancer cells, we knocked down the expression of PKM2 in CD44 ${ }^{+}$CSCs by PKM siRNA (L-006781-00-0005; GE Healthcare Dharmacon, Inc., Lafayette, CO, USA). The control group was transfected with a negative control siRNA (Thermo Fisher Scientific/ Dharmacon). Briefly, cells were seeded in 6-well plates or 4-well chamber slides (for immunostaining), and transient transfections were then performed with DharmaFECT-1 transfection reagent according to THE manufacturer's protocol. The efficiency on PKM2 siRNA knockdown was confirmed by western blot analysis as described below.

Western blot analysis. The expression level of PKM2 in CD44+ CSCs was measured by western blot analysis as previously described (23). Briefly, the total protein was purified from cells, separated using SDS-PAGE gels, and then were transferred to nitrocellulose membranes. After blocking, the membranes were incubated with rabbit polyclonal antibody specific against PKM2 (\#4053S; Cell Signal Technology) or goat monoclonal antibody against $\beta$-actin (Cell Signal Technology), followed by the appropriate horseradish peroxidase-conjugated secondary antibodies. The expression was visualized using an enhanced chemiluminescence detection kit.

Spheroid formation assay. We further examined the characterization of CSCs by spheroid formation assay. Briefly, $1 \times 10^{3} \mathrm{CD}_{4} 4^{+} \mathrm{CSC}$ s were seeded in $60 \mathrm{~mm}$ low cell binding dish (Nunc, Roskilde, Denmark). After 7 days of culture, the formation of spheres was observed under phase-contrast microscopy and the total number of spheroids in each culture dish was counted.

Clonogenic assay. For clonogenic assay, we seeded CD $44^{+}$ CSCs into 6-well plates at a density of 100 cells/well (24). After an overnight incubation, the cells were exposed to the indicated doses of $\gamma$-rays, and the formation of colonies was quantified 7 days after irradiation. Colonies with $>50$ cells were counted under a microscope.

Assessment of anticancer drug sensitivity. To evaluate the anticancer drug sensitivity, cells were seeded in 96-well culture plates at a density of $5 \times 10^{3}$ cells/well and cultured overnight. Cells were then treated with various concentrations of cisplatin (CDDP). After $48 \mathrm{~h}$, cell survival and proliferation was evaluated by the MTT [3-(4,5-dimethylthiazol-2-yl)2,5-diphenyltetrazolium bromide] assay. Briefly, MTT were added to medium for $4 \mathrm{~h}$, and the formation of formazan from MTT in viable cells was stopped by adding lysis buffer $24 \mathrm{~h}$ after the addition of MTT. The absorbance of formazan was measured at $570 \mathrm{~nm}$ using a microplate reader (Thermo Scientific Multiskan FC).

Estimation of the tolerance to FBS and oxygen depletion stresses. To evaluate the resistance of cells to FBS deprivation and hypoxic stress, cells were seeded in 96-well culture plates at a density of $5 \times 10^{3}$ cells/well and maintained in a low FBS $\left(1 \%\right.$ FBS) culture medium or low oxygen condition $\left(1 \% \mathrm{O}_{2}\right)$ for $24 \mathrm{~h}$. The survival and proliferation of cells was then measured by the MTT assay as described above.

Statistical analysis. All results are presented as the means \pm SD. The statistical analysis was done by one-way ANOVA followed by the Bonferroni post hoc test among groups, or by the unpaired t-test between two groups (SPSS II; 
A

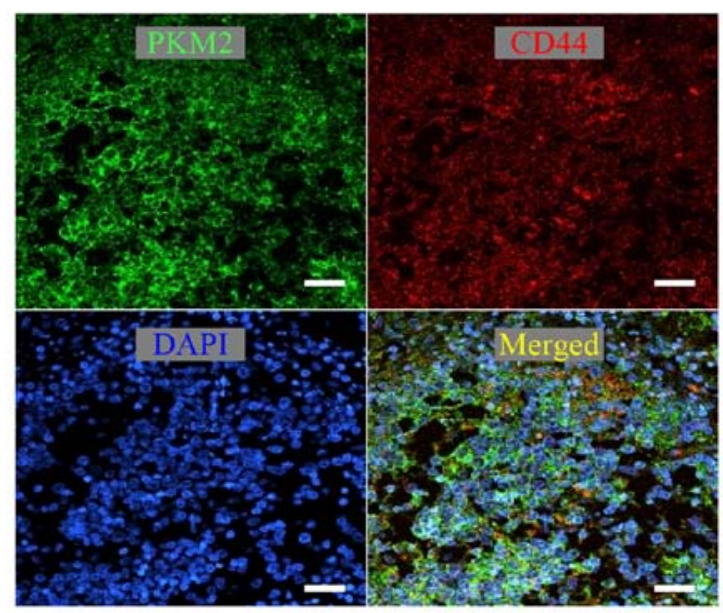

B

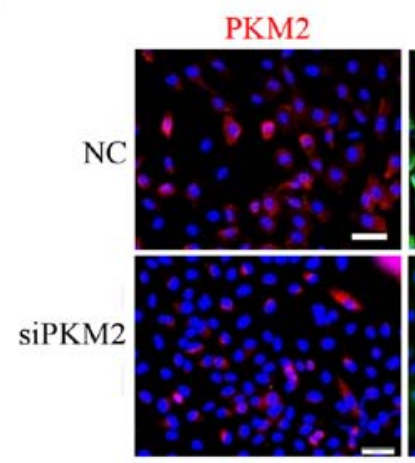

A549 cells

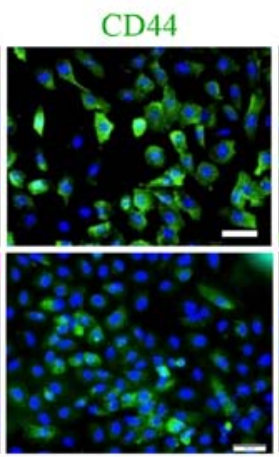

C
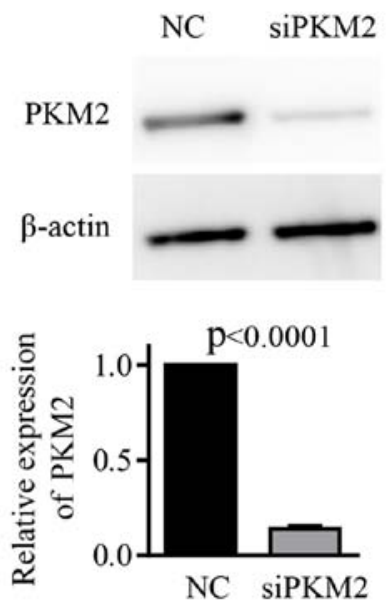

Figure 1. Relationship between the PKM2 and CD44 expression. (A) The expression of PKM2 and CD44 in lung cancer tissue samples from patients. Scale bar, $50 \mu \mathrm{m}$. (B) The expression of PKM2 in CD44 ${ }^{+}$cancer stem cells with or without PKM2 knockdown. Scale bar, $50 \mu \mathrm{m}$. (C) Western blot analysis on PKM2 expression in the CD44+ cancer stem cells with or without PKM2 knockdown. NC, negative control siRNA.

SPSS, Inc., Chicago, IL, USA). Differences were considered significant at $\mathrm{P}<0.05$.

\section{Results}

Co-expression of PKM2 and CD44 in lung cancer cells from surgically resected tissue samples. By using lung cancer tissue samples from patients, we could clearly observe that some lung cancer cells positively expressed CD44 (Fig. 1A). Furthermore, these $\mathrm{CD} 44^{+}$cancer cells were also highly expressed with PKM2 (Fig. 1A), supported the likely relationship between PKM2 expression and cancer stem cell phenotype.

Enhanced expression of PKM2 is closely associated with the phenotype of CSCs. PKM2 was highly expressed in 80\% of the A549 lung cancer cells. Over 70\% of these A549 cells also positively expressed CD44, a marker popularly used for identifying CSCs. We noted that the enhanced expression of PKM2 was specially observed in the cells that also strongly expressed CD44 (Fig. 1B).

To confirm the directly relationship between PKM2 expression and the biological property of CSCs, we purified CD44 ${ }^{+}$ CSCs for further studies. We knocked down the expression of PKM2 in $\mathrm{CD}_{4} 4^{+} \mathrm{CSC}$ s by siRNA, and then observed how the decrease of PKM2 expression could change the phenotype of CD $44^{+}$CSCs. The siRNA knockdown clearly decreased the
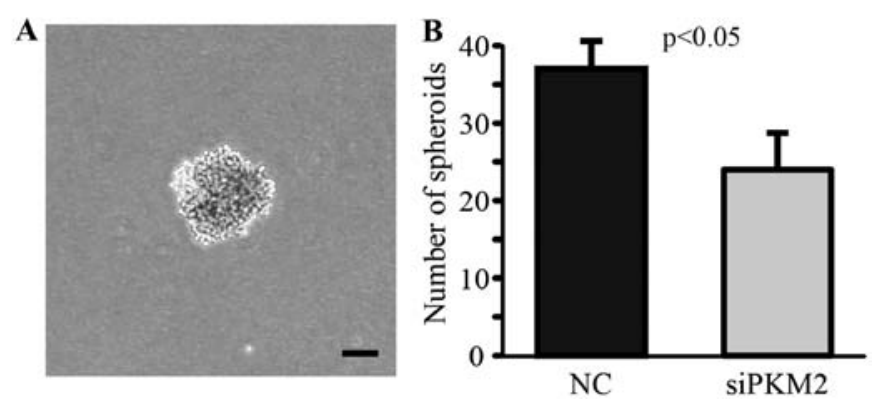

Figure 2. Spheroid formation capacity of $\mathrm{CD} 44^{+}$cancer stem cells. The number of spheroids in each culture dish was counted by microscopy. (A) Representative image of spheroid formation. Scale bar, $100 \mu \mathrm{m}$. (B) The average number of spheroids. Data are presented as mean \pm SD. NC, negative control siRNA.

expression of PKM2 in CD44 ${ }^{+} \mathrm{CSCs}$ (Fig. 1B and C), and also resulted in a slightly decrease in the expression of CD44 in cells (Fig. 1B).

By using the spheroid forming assay, a widely accepted in vitro method for the assessment of cancer stem cell potency, we found that the knockdown of PKM2 significantly decreased the property of $\mathrm{CD} 44^{+} \mathrm{CSCs}$ in forming spheroid in vitro (Fig. 2). These data suggest that the enhanced PKM2 expression directly associates with cancer stem cell phenotype. 

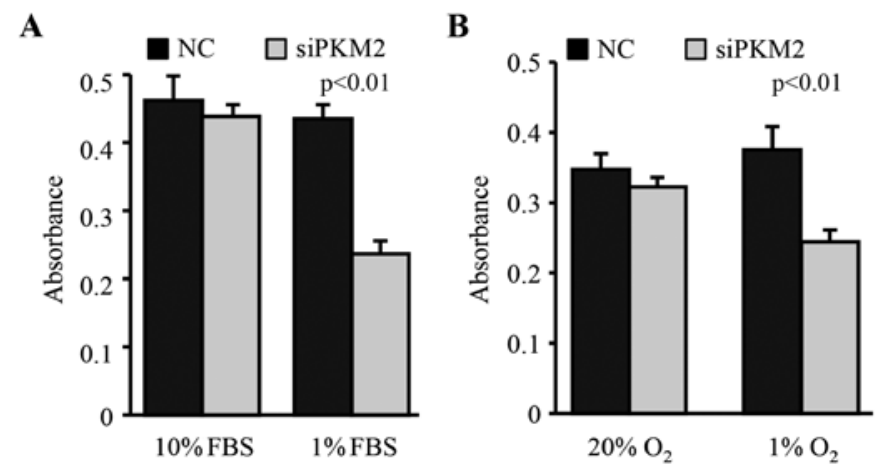

Figure 3. The tolerance of $\mathrm{CD} 44^{+}$cancer stem cells under fetal bovine serum (FBS) deprivation and hypoxic conditions. (A) $\mathrm{CD} 44^{+}$cancer stem cells with or without the knockdown of PKM2 were cultured in medium supplemented with 1 or $10 \%$ FBS, and the cell survival was evaluated by MTT assay $24 \mathrm{~h}$ after culture. (B) $\mathrm{CD} 44^{+}$cancer stem cells with or without the knockdown of PKM2 were maintained in 1 or $20 \%$ oxygen incubators and the cell survival was evaluated by MTT assay $24 \mathrm{~h}$ after culture. Data are presented as mean \pm SD. NC, negative control siRNA.
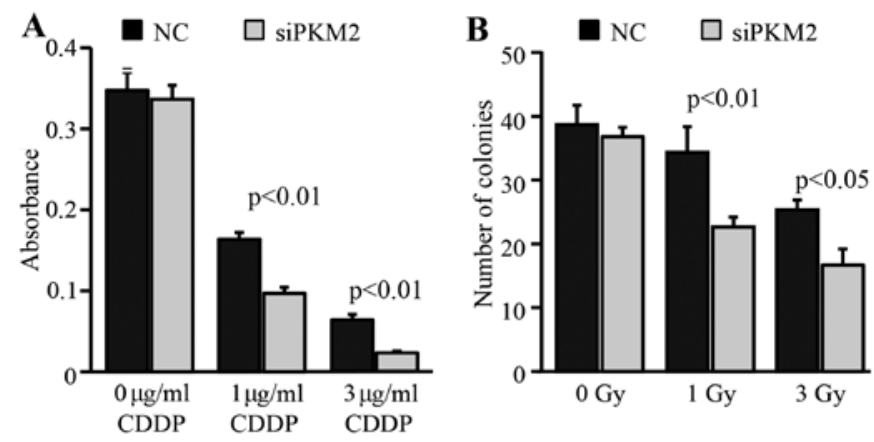

Figure 4 . The resistance of $\mathrm{CD} 44^{+}$cancer stem cells to chemotherapy and radiotherapy. (A) $\mathrm{CD} 44^{+}$cancer stem cells with or without the knockdown of PKM2 were exposed to different concentrations of cisplatin (CDDP) in medium, and the cell survival was evaluated by MTT assay $48 \mathrm{~h}$ after CDDP treatment. (B) $\mathrm{CD} 44^{+}$cancer stem cells with or without the knockdown of PKM2 were exposed to different doses of $\gamma$-ray, and the formation of colonies from the surviving cancer cells was counted 7 days after $\gamma$-ray exposure. Data are presented as mean \pm SD. Scale bar, $200 \mu \mathrm{m}$. NC, negative control siRNA.

Enhanced PKM2 expression contributes to the stress resistance of CSCs. To test how the enhanced PKM2 expression in $\mathrm{CD} 44^{+} \mathrm{CSCs}$ contributes to resistant to stresses, we exposed cells to FBS deprivation and hypoxic conditions. We found that the downregulation of PKM2 in CD44 ${ }^{+}$CSCs did not change their survival in medium supplemented with $10 \%$ FBS and under $20 \%$ oxygen condition (Fig. 3). However, the knockdown of PKM2 by siRNA significantly decreased the survival of $\mathrm{CD}_{4} 4^{+} \mathrm{CSC}$ under FBS deprivation (1\% FBS) and low oxygen $\left(1 \% \mathrm{O}_{2}\right)$ conditions (Fig. 3).

Enhanced PKM2 expression contributes to the therapeutic resistance of CSCs. We also examined how the enhanced expression of PKM2 in CD44 ${ }^{+} \mathrm{CSC}$ contributes to resistance to chemotherapy and radiotherapy. The knockdown of PKM2 expression in $\mathrm{CD}_{4} 4^{+} \mathrm{CSC}$ significantly decreased their survival under different concentrations of CDDP (Fig. 4A). Similarly, clonogenic assay showed that the knockdown of PKM2 expression also significantly decreased the colony formation capacity of $\mathrm{CD} 44^{+} \mathrm{CSC}$ after exposure to 1 or $3 \mathrm{~Gy}$ $\gamma$-ray (Fig. 4B).

\section{Discussion}

PKM2 is generally known to be expressed at high levels in tumors, which functionally contribute to the rapidly growth of cancer cells even under a relative hypoxic microenvironment (3-6,25). However, PKM2 is not necessary for tumor cell proliferation, and heterozygous PKM2 mutations have been found in human tumors (26). It has also been implied that the inactive state of PKM2 is associated with the proliferating cell population within tumors, but non-proliferating tumor cells require active pyruvate kinase (26). This suggests that the variable of PKM2 expression supports the different metabolic requirements of proliferating and non-proliferating tumor cells. Considering the heterogeneity of tumor cells $(10,11)$, the relative quiescent state of cancer stem cells may well match to these non-proliferating tumor cells with high pyruvate kinase expression. Actually, our data from both clinical lung cancer tissue samples and a human lung cancer cell line have clearly shown an enhanced expression of PKM2 in these cancer cells that positively expressed CD44, a popular marker for CSCs in various types of cancers.

To further confirm the role of PKM2 on the biological property of cancer stem cells, we knocked down the PKM2 expression in $\mathrm{CD} 44^{+} \mathrm{CSCs}$. In agreement with a previous study (27), the PKM2 knockdown in CD $44^{+} \mathrm{CSC}$ s resulted in a modest impairment of cell proliferation under general culture conditions. Cell apoptosis is rarely observed in these CD44 CSCs by TUNEL staining, thus, we did not regularly evaluate cell apoptosis in this study. Notably, the PKM2 knockdown in $\mathrm{CD} 44^{+} \mathrm{CSC}$ significantly decreased the capacity of spheroids formation in vitro and the resistant to FBS deprivation and hypoxic stress, but significantly increased the sensitivity to chemotherapy and radiotherapy. All of these data confirmed the direct relationship between the enhanced expression of PKM2 and the biological property of cancer stem cells. As PKM1 and PKM2 are different splicing products of PKM gene (exon 9 for PKM1 and exon 10 for PKM2) (28), it is impossible to specifically knock down PKM2. Although cancer cells are known to mainly express PKM2, the transfection with PKM siRNA in the present study may also change the expression of PKM1 and probably results in some other off-target effects.

The expression of CD44 in cancer stem cells has been previously demonstrated to associate with the resistance to oxidative stress and chemotherapy (29). PKM2 expression in cancer cells is also well known to contribute to tumor progression and therapeutic resistance (3-7,30). A recent study has reported that the ablation of CD44 expression in hypoxic cancer cells significantly increases the expression of PKM2 but decreases glucose uptake, which results in an enhanced sensitivity to anticancer drugs (21). In the present study, we simply used CD44 as a marker for the identification/purification of cancer stem cells. An enhanced expression of PKM2 was observed in these CD $44^{+} \mathrm{CSCs}$, but the role of CD44 expression on the resistance to drugs was not investigated. Therefore, the direct regulatory relationship between PKM2 and CD44 is still questionable. This study is largely limited by 
using a single cell line. We attempted to isolate $\mathrm{CD} 44^{+}$cells from several other lung cancer cell lines, such as NCI-H1975, NCI-H1299 and NCI-H1437 cells. However, the purified $\mathrm{CD} 44^{+}$cells from these cell lines showed very poor stability on the expression of CD44 during the culture process. Further studies in other cancer cell lines will be needed to confirm our findings.

Although the clear relationship between the enhanced expression of PKM2 and the biological property of cancer stem cells, it is unclear how the PKM2 expression facilitates the biological property of cancer stem cells. Beyond the function in regulating anabolic metabolism, PKM2 is also known to serve as transcriptional co-activators (4-7). A recent study has demonstrated that the stimulation of epithelial-mesenchymal transition results in the nuclear translocation of PKM2 in colon cancer cells (31). Furthermore, it has been found that the phosphorylated PKM2 can translocate into the nucleus to serve as a co-activator of $\beta$-catenin to induce c-Myc expression (32). As the expression of $\mathrm{c}-\mathrm{Myc}$ and $\beta$-catenin has been demonstrated to associate with normal tissue stem cells, pluripotent stem cells, and cancer stem cells (33-35), it is possible that PKM2 facilitates the biological property of cancer stem cells through the induction of $\beta$-catenin and c-Myc.

The data from the present study clearly indicate that the enhanced expression of PKM2 in lung cancer cells likely associated with the biological properties of cancer stem cells, including the resistant to various stresses and therapies. Therefore, selective targeting of PKM2 may change the biological property of cancer stem cells, which provides a new strategy for treatment of cancer.

\section{Acknowledgements}

The present study was supported by the Jiangxi Province's Program of the Preponderant Team Building in Science and Technology Innovation (no. 20161BCB24011), the Jiangxi Province Sailing Project, the National Natural Science Foundation of China (no. 81560382); and a grant-in-aid from the Ministry of Education, Science, Sports, Culture and Technology, Japan.

\section{References}

1. Vander Heiden MG, Lunt SY, Dayton TL, Fiske BP, Israelsen WJ, Mattaini KR, Vokes NI, Stephanopoulos G, Cantley LC, Metallo CM, et al: Metabolic pathway alterations that support cell proliferation. Cold Spring Harb Symp Quant Biol 76 : 325-334, 2011

2. Christensen DR, Calder PC and Houghton FD: GLUT3 and PKM2 regulate OCT4 expression and support the hypoxic culture of human embryonic stem cells. Sci Rep 5: 17500, 2015.

3. Iqbal MA, Gupta V, Gopinath P, Mazurek S and Bamezai RN: Pyruvate kinase M2 and cancer: An updated assessment. FEBS Lett 588: 2685-2692, 2014.

4. Tamada M, Suematsu M and Saya H: Pyruvate kinase M2. Multiple faces for conferring benefits on cancer cells. Clin Cancer Res 18: 5554-5561, 2012.

5. Luo W and Semenza GL: Emerging roles of PKM2 in cell metabolism and cancer progression. Trends Endocrinol Metab 23: 560-566, 2012

6. Jiang Y, Li X, Yang W, Hawke DH, Zheng Y, Xia Y, Aldape K, Wei C, Guo F, Chen Y, et al: PKM2 regulates chromosome segregation and mitosis progression of tumor cells. Mol Cell 53: 75-87, 2014.
7. Li Z, Yang $\mathrm{P}$ and $\mathrm{Li}$ Z: The multifaceted regulation and functions of PKM2 in tumor progression. Biochim Biophys Acta 1846: 285-296, 2014.

8. Li SL, Ye F, Cai WJ, Hu HD, Hu P, Ren H, Zhu FF and Zhang DZ: Quantitative proteome analysis of multidrug resistance in human ovarian cancer cell line. J Cell Biochem 109: 625-633, 2010.

9. Wang Y, Zhang X, Zhang Y, Zhu Y, Yuan C, Qi B, Zhang W, Wang D, Ding X, Wu H, et al: Overexpression of pyruvate kinase M2 associates with aggressive clinicopathological features and unfavorable prognosis in oral squamous cell carcinoma. Cancer Biol Ther 16: 839-845, 2015.

10. Meacham CE and Morrison SJ: Tumour heterogeneity and cancer cell plasticity. Nature 501: 328-337, 2013.

11. Valent P, Bonnet D, Wöhrer S, Andreeff M, Copland M, Chomienne C and Eaves C: Heterogeneity of neoplastic stem cells: Theoretical, functional, and clinical implications. Cancer Res 73: 1037-1045, 2013.

12. Islam F, Gopalan V, Smith RA and Lam AK: Translational potential of cancer stem cells: A review of the detection of cancer stem cells and their roles in cancer recurrence and cancer treatment. Exp Cell Res 335: 135-147, 2015.

13. Tomasetti $\mathrm{C}$ and Vogelstein B: Cancer etiology. Variation in cancer risk among tissues can be explained by the number of stem cell divisions. Science 347: 78-81, 2015.

14. Visvader JE: Cells of origin in cancer. Nature 469: 314-322, 2011.

15. Woodward WA and Sulman EP: Cancer stem cells: Markers or biomarkers? Cancer Metastasis Rev 27: 459-470, 2008.

16. $\mathrm{Wu} \mathrm{Y}$ and $\mathrm{Wu}$ PY: $\mathrm{CD} 133$ as a marker for cancer stem cells: Progresses and concerns. Stem Cells Dev 18: 1127-1134, 2009.

17. Leung EL, Fiscus RR, Tung JW, Tin VP, Cheng LC, Sihoe AD, Fink LM, Ma Y and Wong MP: Non-small cell lung cancer cells expressing CD44 are enriched for stem cell-like properties. PLoS One 5: e14062, 2010.

18. Plaks V, Kong N and Werb Z: The cancer stem cell niche: How essential is the niche in regulating stemness of tumor cells? Cell Stem Cell 16: 225-238, 2015.

19. Suda T, Takubo K and Semenza GL: Metabolic regulation of hematopoietic stem cells in the hypoxic niche. Cell Stem Cell 9: 298-310, 2011.

20. Bertolini G, Roz L, Perego P, Tortoreto M, Fontanella E, Gatti L, Pratesi G, Fabbri A, Andriani F, Tinelli S, et al: Highly tumorigenic lung cancer $\mathrm{CD} 133^{+}$cells display stem-like features and are spared by cisplatin treatment. Proc Natl Acad Sci USA 106: 16281-16286, 2009.

21. Tamada M, Nagano O, Tateyama S, Ohmura M, Yae T, Ishimoto T, Sugihara E, Onishi N, Yamamoto T, Yanagawa H, et al: Modulation of glucose metabolism by CD44 contributes to antioxidant status and drug resistance in cancer cells. Cancer Res 72: 1438-1448, 2012.

22. Guo CY, Luo L, Urata Y, Goto S, Huang WJ, Takamura S, Hayashi F, Doi H, Kitajima Y, Ono Y, et al: Sensitivity and dose dependency of radiation-induced injury in hematopoietic stem/ progenitor cells in mice. Sci Rep 5: 8055, 2015.

23. Li TS, Cheng K, Lee ST, Matsushita S, Davis D, Malliaras K, Zhang Y, Matsushita N, Smith RR and Marbán E: Cardiospheres recapitulate a niche-like microenvironment rich in stemness and cell-matrix interactions, rationalizing their enhanced functional potency for myocardial repair. Stem Cells 28: 2088-2098, 2010.

24. Yan C, Luo L, Goto S, Urata Y, Guo CY, Doi H, Kitazato K and Li TS: Enhanced autophagy in colorectal cancer stem cells does not contribute to radio-resistance. Oncotarget 7: 45112-45121, 2016.

25. Christofk HR, Vander Heiden MG, Harris MH, Ramanathan A, Gerszten RE, Wei R, Fleming MD, Schreiber SL and Cantley LC: The M2 splice isoform of pyruvate kinase is important for cancer metabolism and tumour growth. Nature 452: 230-233, 2008.

26. Israelsen WJ, Dayton TL, Davidson SM, Fiske BP, Hosios AM, Bellinger G, Li J, Yu Y, Sasaki M, Horner JW, et al: PKM2 isoform-specific deletion reveals a differential requirement for pyruvate kinase in tumor cells. Cell 155: 397-409, 2013.

27. Cortés-Cros M, Hemmerlin C, Ferretti S, Zhang J, Gounarides JS, Yin H, Muller A, Haberkorn A, Chene P, Sellers WR, et al: M2 isoform of pyruvate kinase is dispensable for tumor maintenance and growth. Proc Natl Acad Sci USA 110: 489-494, 2013. 
28. Dombrauckas JD, Santarsiero BD and Mesecar AD: Structural basis for tumor pyruvate kinase M2 allosteric regulation and catalysis. Biochemistry 44: 9417-9429, 2005.

29. Ishimoto T, Nagano O, Yae T, Tamada M, Motohara T, Oshima H, Oshima M, Ikeda T, Asaba R, Yagi H, et al: CD44 variant regulates redox status in cancer cells by stabilizing the $\mathrm{xCT}$ subunit of system $\mathrm{xc}(-)$ and thereby promotes tumor growth Cancer Cell 19: 387-400, 2011.

30. Papadaki C, Sfakianaki M,Lagoudaki E, Giagkas G, Ioannidis G, Trypaki M, Tsakalaki E, Voutsina A, Koutsopoulos A, Mavroudis D, et al: PKM2 as a biomarker for chemosensitivity to front-line platinum-based chemotherapy in patients with metastatic non-small-cell lung cancer. Br J Cancer 111: 1757-1764, 2014.

31. Hamabe A, Konno M, Tanuma N, Shima H, Tsunekuni K, Kawamoto K, Nishida N, Koseki J, Mimori K, Gotoh N, et al: Role of pyruvate kinase $\mathrm{M} 2$ in transcriptional regulation leading to epithelial-mesenchymal transition. Proc Natl Acad Sci USA 111: 15526-15531, 2014.
32. Yang W, Zheng Y, Xia Y, Ji H, Chen X, Guo F, Lyssiotis CA, Aldape K, Cantley LC and Lu Z: ERK1/2-dependent phosphorylation and nuclear translocation of PKM2 promotes the Warburg effect. Nat Cell Biol 14: 1295-1304, 2012.

33. Benoit YD, Guezguez B, Boyd AL and Bhatia M: Molecular pathways: Epigenetic modulation of Wnt-glycogen synthase kinase-3 signaling to target human cancer stem cells. Clin Cancer Res 20: 5372-5378, 2014.

34. Chappell J and Dalton S: Roles for MYC in the establishment and maintenance of pluripotency. Cold Spring Harb Perspect Med 3: a014381, 2013.

35. Holland JD, Klaus A, Garratt AN and Birchmeier W: Wnt signaling in stem and cancer stem cells. Curr Opin Cell Biol 25: 254-264, 2013 